\title{
SIOP/COG/NWTSG y-Stage II
}

National Cancer Institute

\section{Source}

National Cancer Institute. SIOP/COG/NWTSGy-Stage II. NCI Thesaurus. Code C140304.

The assessment of stage III Wilms' tumor was performed after neoadjuvant therapy was given. 